\title{
Hétérozygotie et durée de développement chez Drosophila melanogaster
}

\author{
P. GIRARD \\ Université de Paris 7, Laboratoire de Génétique des Populations \\ U.A. C.N.R.S. 693, Tour 42-43, 2, place Jussieu, F 75005 Paris
}

\section{Résumé}

Des souches polymorphiques pour un, deux ou six locus enzymatiques (Acph, Adlh, $\alpha-G p d h, E s t-6, E s t-C$ et $P g m$ ) ont été extraites de 3 populations naturelles françaises de Drosophila melanogaster. étudiée.

La relation entre la durée de développement totale et le génotype individuel a été

Les résultats sont les suivants :

1) La durée de développement est très différente d'un génotype à l'autre.

2) Les individus hétérozygotes ont une durée de développement systématiquement plus courte que les homozygotes.

3) Les différences extrêmes entre les durées de développement sont plus grandes dans le cas des ségrégations à 2 locus que dans les ségrégations simples correspondantes.

4) La ségrégation à 6 locus démontre qu'il existe une corrélation négative entre la durée de développement et le degré d'hétérozygotie.

5) Cependant, la variabilité de chaque classe d'hétérozygotie ne peut s'expliquer uniquement par le nombre de génotypes distincts de chaque classe d'hétérozygotie.

Ces résultats sont en bon accord avec l'existence d'une over-dominance systématique des locus enzymatiques, sans exclure pour autant l'existence possible de gènes délétères.

Mots clés : Drosophila melanogaster, polymorphisme enzymatique, hétérozygotie, durée de développement, hétérosis.

\section{Summary}

Heterozygosity and developmental time in Drosophila melanogaster

Individuals extracted from 3 natural populations of Drosophila melanogaster have been used to make strains polymorphic for 1,2 or 6 loci $(A c p h, A d h, \alpha-G p d h, E s t-6, E s t-C$, and Pgm).

A relation between development time and genotype has been looked for. The results are the following :

1) Development time is highly different from one genotype to another.

2) Heterozygous individuals have a shorter development time than homozygous. 
3) The extreme differences are greater in case of 2 loci segregation than in case of one.

4) The 6 loci segregation demonstrate a significant negative correlation between development time and heterozygosity.

5) Therefore, the large variability in each class of heterozygosity cannot be explained only by the number of different genotypes in each class.

Our results are in good agreement with a systematic overdominance of heterozygous enzymatic loci, a hypothesis that does not exclude the existence of some deleterious genes.

Key words : Drosophila melanogaster, enzymatic polymorphism, heterozygosity, developmental time, heterosis.

\section{Introduction}

L'avantage de l'état hétérozygote est, depuis les débuts de la génétique des populations, considéré comme un des facteurs majeurs de maintien du polymorphisme (Dobzhansky, 1937 ; TeIssier, 1942 ; Fisher, 1949 ; Boesiger, 1962). Cependant, les démonstrations expérimentales rigoureuses de cet avantage des hétérozygotes sont rares.

L'étude des populations naturelles a permis la mise en évidence de corrélations entre l'hétérozygotie et les évaluations de différents constituants de la valeur adaptative (SchAAL \& LeviN, 1976 ; Soule, 1979 ; Zouros et al., 1980), mais le mécanisme du phénomène n'est pas encore clair. En effet, ces expériences ne permettent pas de différencier ce qui, dans le désavantage des homozygotes, est dû à la présence de gènes récessifs défavorables, ou à une diminution du degré d'hétérozygotie du génome (Sved \& Ayala, 1970 ; Lewontin, 1974).

Dans ce contexte, l'une des premières questions à résoudre est celle de l'importance relative de l'hétérozygotie à chaque locus dans la variation de chacun des constituants de la valeur adaptative.

Ainsi certains auteurs ont recherché l'existence de corrélations entre le degré d'hétérozygotie et l'évaluation de certains caractères quantitatifs (CHAISson et al., 1976 ; Stalker, 1976 ; Mitton, 1978 ; Singh \& Zouros, 1978 ; Mitton \& Grant, 1980).

Un nombre croissant de travaux dans ce domaine concerne l'étude de la vitesse de développement, composante capitale de la valeur adaptative (LEWONTIN, 1965 ; SchaAl \& Levin, 1976 ; Girard, 1980).

La plupart d'entre eux ont été réalisés sur des populations supposées, ou non, à l'équilibre. Mais compte tenu du nombre de facteurs de variation susceptibles d'intervenir dans ce type d'expérience, nous lui avons préféré l'étude de ségrégations individuelles à un ou plusieurs locus simultanément.

Cette technique permet en effet un élargissement du champ de variation de l'hétérozygotie des locus considérés par rapport aux populations naturelles (SvED et al., 1967), ce qui augmente les chances de mise en évidence d'une corrélation significative, noyée, dans les populations naturelles, par le "bruit » dû à l'immense variabilité du génome (Soule, 1979). 
Nous nous sommes donc proposé de préciser, à partir de croisements individuels, la liaison entre l'hétérozygotie observée et la durée de développement de l'œuf à l'adulte, sans exclure $a$ priori l'hypothèse d'un déterminisme spécifique.

\section{Matériel et méthodes}

\section{A. Matériel biologique}

Les individus utilisés en tant que fondateurs dans les expériences provenaient de populations naturelles françaises de Drosophila melanogaster précédemment analysées (GirARD, 1976 ; Girard et al., 1977).

\section{Les locus}

Six locus enzymatiques ont été sélectionnés parmi les locus polymorphes de fréquence allélique égale ou supérieure à 0,05 . La facilité et la rapidité des techniques de révélation enzymatique a servi de critère de choix.

Le tableau 1 regroupe ces locus avec leurs caractéristiques spécifiques. Deux des locus sont situés sur le chromosome II à 32,3 centimorgans l'un de l'autre ; les quatre autres sont sur le chromosome III, trois d'entre eux étant étroitement liés. Pour chacun de ces locus, seuls les 2 allèles les plus fréquents ont été retenus. Ils sont symbolisés par les lettres $F$ et $S$ correspondant aux dénominations Fast et $S l o w$ relatives à la position électrophorétique des allélomorphes respectifs. Il existe donc 3 génotypes possibles par locus : FF, $F S$ et $S S$, que seront symbolisés respectivement par $: F$., $H$. et $S$. ; un individu hétérozygote pour les 3 premiers locus et homozygote de la forme Slow pour le $4^{\mathrm{e}}$ sera noté : H.H.H.S.

\section{TABLEAU 1}

Les locus étudiés.

The loci studied.

\begin{tabular}{|c|c|c|c|}
\hline Locus & Symbole & Localisation & Références \\
\hline $\begin{array}{l}\alpha \text {-glycérophosphate deshy- } \\
\text { drogénase } \ldots \ldots \ldots \ldots\end{array}$ & $\alpha-G p d h$ & II $; 17,8$ & E.H. Grell, 1967 \\
\hline Alcool deshydrogénase .. & Adh & II $; 50,1$ & $\begin{array}{l}\text { E.H. GRELL, K.B. JACOB- } \\
\text { SON, J.B., MURPHY, } 1965\end{array}$ \\
\hline Estérase-6 & Est-6 & III ; 36,8 & T.R.F. WRIGHT, 1963 \\
\hline Phosphoglucomutase & $\mathrm{Pgm}$ & III ; 43,4 & J.P. HJORTH, 1970 \\
\hline Estérase-C . . . . & Est-C & III ; 47,6 & $\begin{array}{l}\text { L. BECKMAN, M. JoHNSON, } \\
1964\end{array}$ \\
\hline Phosphatase acide & Acph-1 & III ; 101,4 & R. MAC INTYRE, 1966 \\
\hline
\end{tabular}




\section{Conditions d'élevage}

Les milieux d'élevage sont tels que la compétition alimentaire soit réduite au maximum ; ainsi pourra-t-on supposer que les différences individuelles dans les durées de développement des génotypes sont dues essentiellement à la structure génétique globale des individus, l'influence des conditions alimentaires n'intervenant que très secondairement.

Les expériences ont été faites en principe à une température de $25^{\circ} \mathrm{C}$; mais, compte tenu de la longueur de la période expérimentale qui s'est étalée sur plusieurs années, certaines différences sont intervenues d'une expérience à l'autre, différences dont l'incidence sera discutée avec les résultats du tableau 2.

\section{B. Protocoles expérimentaux}

Des croisements individuels entre hétérozygotes pour un ou plusieurs locus, ou entre un hétérozygote et un homozygote ont été réalisés ; ainsi obtient-on, en $1^{\text {re géné- }}$ ration, le nombre maximal de génotypes distincts, et avec les fréquences les plus élevées.

\section{Expériences préliminaires}

A partir de plusieurs populations naturelles françaises, Saint-Cézaire-sur-Siagne 1976 et 1978 (S.C.S. 1 et S.C.S. 2) et Remoulins 1976 (Rem), des croisements individuels entre porteurs de génotypes variés (F., H. ou S.) ont été réalisés pour différents locus selon le protocole suivant : des couples de la population naturelle choisie sont placés pour 24 heures dans des tubes contenant un milieu nutritif riche à base de farine de maïs et ensemencé de levure fraîche. Le génotype des parents est déterminé par électrophorèse et seuls les couples comportant au moins un individu hétérozygote sont retenus. Le génome de ces individus reste donc équilibré.

Les descendants de $1^{\text {re }}$ génération sont prélevés jour par jour (ou par fraction de jour) durant la phase d'émergence, dans des conditions strictement identiques pour une même expérience (même heure, même séquence d'opérations ordonnées).

La constitution génétique des descendants de $1^{\text {re }}$ génération est déterminée ensuite en totalité par électrophorèse. Selon le nombre de locus où l'on constate une ségrégation en F1, celle-ci est appelée monofactorielle (pour un locus), bifactorielle (pour 2), hexafactorielle (pour 6 locus). Les expériences préliminaires n'ont porté que sur des ségrégations mono- et bifactorielles.

\section{Expérience hexafactorielle}

a) Constitution des souches : à partir de la population naturelle Remoulins 76, deux souches homozygotes pour chacun des 6 locus, opposées et complémentaires quant à leur constitution génétique, ont été isolées : la souche $S 1$ est de génotype : F.S.S.S.F.F. et la souche $S 2$ : S.F.F.F.S.S., les locus étant pris dans l'ordre suivant : a-Gpdh, Adh, Est-6, Pgm, Est-C et Acph-1.

b) Les croisements : des croisements individuels entre hétérozygotes $S 1 / S 2$ ont été réalisés dans les mêmes conditions d'élevage que précédemment : les imagos, 
placés pendant 24 heures dans un milieu riche, sont changés de tube tous les jours pendant 3 jours, fournissant 4 échantillons d'œufs pondus chacun sur un intervalle de 24 heures. La totalité des individus de première génération est, par lots journaliers et par jour de ponte, analysée par électrophorèse.

\section{Techniques d'électrophorèse}

Les techniques d'électrophorèse sur gel d'amidon et les systèmes de révélation enzymatique utilisés ont été décrits en détail dans une précédente publication (GIRARd et al., 1977).

\section{Variables étudiées}

La durée moyenne de développement $\mathrm{D}$ de chaque génotype a été calculée. Les valeurs de la variable aléatoire $\mathrm{D}$ sont rapportées à une origine zéro correspondant au jour précédant l'émergence des premiers descendants de $1^{\text {re }}$ génération. La valeur de la différence entre le moment de la copulation et cette origine zéro est variable d'une ségrégation à l'autre en fonction de plusieurs paramètres dont l'étude ne fait pas partie de ce travail. Les valeurs numériques des durées de développement sont donc relatives et calculées à partir de la valeur 1 correspondant au premier jour d'émergence.

L'estimation de l'hétérozygotie $H$ d'un individu est obtenue directement par le nombre de locus de cet individu à l'état hétérozygote, $(\mathrm{H}: 0,1,2, \ldots, \mathrm{p}), \mathrm{p}$ étant le nombre de locus examinés.

Dans les expériences monofactorielles ou bifactorielles, l'appartenance d'un individu à un génotype donné a été considérée comme un caractère qualitatif. Par contre, dans l'expérience hexafactorielle, nous avons défini 2 critères de classification sur l'ensemble des individus : l'appartenance à une classe d'hétérozygotie $\mathbf{H}$ et l'appartenance à une classe de durée de développement D. Selon les questions soulevées, ces 2 critères ont été considérés indépendamment l'un de l'autre, soit comme une variable qualitative, soit comme une variable quantitative.

\section{E. Techniques d'analyse statistique}

Les tests d'homogénéité utilisés sont le test du rapport de vraisemblance (G-test) de Woolf (1956), qui est un test " grands échantillons » et le test d'Haldane-Dawson qui convient dans tous les cas. Enfin, pour comparer 2 tables de contingences, on a utilisé le test des matrices de transition de HiLton (1957).

Les analyses de variance à un facteur et les calculs de corrélation ont été réalisés selon les méthodes classiques (DAGNELIE, 1975). 


\section{Résultats}

\section{A. Expériences préliminaires}

(ségrégations mono- et bifactorielles)

1. Ségrégations monofactorielles : l'analyse des ségrégations monofactorielles a donné des distributions de durée de développement parfois assez éloignées d'une répartition gaussienne, et seules les distributions correspondant aux conditions de l'analyse de la variance ont été considérées ici (normalité et homogénéité des variances acceptables au niveau 0,05) (tableau 2).

\section{TABI.EAU 2}

Valeurs relatives des durées moyennes de développement $D$ des génotypes $F$, H. et S. pour 5 locus et dans 3 populations naturelles françaises : S.C.S. 1, S.C.S. 2 et Rem.

Relative values of developmental time $D$ of $F$., $H$. and $S$. genotypes, for 5 loci in 3 natural populations : S.C.S. 1, S.C.S. 2 and Rem.

\begin{tabular}{|c|c|c|c|c|c|c|c|c|c|}
\hline \multicolumn{10}{|c|}{ Ségrégations indépendantes } \\
\hline \multirow{2}{*}{ Population } & \multirow{2}{*}{ Locus } & \multicolumn{3}{|c|}{ Durées moyennes } & \multicolumn{5}{|c|}{ Effectifs génotypiques } \\
\hline & & F. & H. & S. & F. & H. & S. & $\mathbf{N}$ & Test $\mathbf{F}$ \\
\hline $\begin{array}{lll}\text { S.C.S. } & 1 & \ldots \\
\text { S.C.S. } & 1 & \ldots \\
\text { S.C.S. } & 1 & \ldots \\
\text { S.C.S. } & 1 & \ldots\end{array}$ & $\begin{array}{l}\text { Adh } \\
\text { Adh } \\
\text { Adh } \\
\text { Adh }\end{array}$ & $\begin{array}{l}7,69 \\
7,43 \\
7,51 \\
6,59\end{array}$ & $\begin{array}{l}7,21 \\
7,62 \\
7,25 \\
6,97\end{array}$ & $\begin{array}{l}7,59 \\
7,45 \\
6,69 \\
6,47\end{array}$ & $\begin{array}{l}24 \\
44 \\
30 \\
39\end{array}$ & $\begin{array}{l}48 \\
77 \\
60 \\
61\end{array}$ & $\begin{array}{l}24 \\
20 \\
30 \\
19\end{array}$ & $\begin{array}{r}96 \\
141 \\
120 \\
119\end{array}$ & $\begin{array}{l}\text { N.S. } \\
\text { N.S. } \\
\text { N.S. } \\
\text { N.S. }\end{array}$ \\
\hline $\begin{array}{lll}\text { S.C.S. } & 1 & \ldots \\
\text { S.C.S. } & 1 & \ldots\end{array}$ & $\begin{array}{l}\alpha-G p d h \\
\alpha-G p d h\end{array}$ & 11,33 & $\begin{array}{l}9,12 \\
8,93\end{array}$ & $\begin{array}{l}9,27 \\
7,79\end{array}$ & 12 & $\begin{array}{l}83 \\
80\end{array}$ & $\begin{array}{l}11 \\
42\end{array}$ & $\begin{array}{l}106 \\
122\end{array}$ & $\begin{array}{l}\text { N.S. } \\
\text { N.S. }\end{array}$ \\
\hline $\begin{array}{lll}\text { S.C.S. } & 1 & \ldots \\
\text { S.C.S. } & 1 & \ldots \\
\text { S.C.S. } & 1 & \ldots\end{array}$ & $\begin{array}{l}\text { Est-C } \\
\text { Est-C } \\
\text { Est-C }\end{array}$ & $\begin{array}{l}5,89 \\
4,23 \\
5,55\end{array}$ & $\begin{array}{l}4,84 \\
3,91 \\
7,36\end{array}$ & - & $\begin{array}{l}18 \\
31 \\
20\end{array}$ & $\begin{array}{l}25 \\
45 \\
36\end{array}$ & - & $\begin{array}{l}43 \\
76 \\
56\end{array}$ & $\underset{*}{*}$ N.S. \\
\hline $\begin{array}{lll}\text { S.C.S. } & 2 & \ldots \\
\text { S.C.S. } & 2 & \ldots \\
\text { S.C.S. } & 2 & \ldots \\
\text { S.C.S. } & 2 & \ldots \\
\text { S.C.S. } & 2 & \ldots \\
\text { S.C.S. } & 2 & \ldots \\
\text { S.C.S. } & 2 & \ldots \\
\text { S.C.S. } & 2 & \ldots\end{array}$ & $\begin{array}{l}\alpha-G p d h \\
\alpha-G p d h \\
\alpha-G p d h \\
\alpha-G p d h \\
\alpha-G p d h \\
\alpha-G p d h \\
\alpha-G p d h \\
\alpha-G p d h\end{array}$ & $\begin{array}{l}- \\
- \\
- \\
-\end{array}$ & $\begin{array}{l}3,10 \\
2,70 \\
3,74 \\
2,87 \\
4,20 \\
4,64 \\
5,03 \\
1,62\end{array}$ & $\begin{array}{l}3,04 \\
2,61 \\
3,16 \\
3,14 \\
4,58 \\
4,88 \\
4,85 \\
2,18\end{array}$ & $\begin{array}{l}- \\
- \\
- \\
-\end{array}$ & $\begin{array}{l}20 \\
44 \\
39 \\
23 \\
39 \\
25 \\
34 \\
21\end{array}$ & $\begin{array}{l}23 \\
36 \\
31 \\
22 \\
33 \\
24 \\
26 \\
17\end{array}$ & $\begin{array}{l}43 \\
80 \\
70 \\
45 \\
72 \\
49 \\
60 \\
38\end{array}$ & $\begin{array}{c}\text { N.S. } \\
\text { N.S. } \\
* \\
\text { N.S. } \\
\text { N.S. } \\
\text { N.S. } \\
\text { N.S. } \\
\text { N.S. }\end{array}$ \\
\hline S.C.S. $2 \ldots$ & Est-C & 2,63 & 3,27 & - & 19 & 26 & - & 45 & $* *$ \\
\hline S.C.S. $2 \ldots$ & Pgm & 3,68 & 3,00 & - & 28 & 23 & - & 51 & $*$ \\
\hline 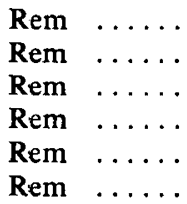 & $\begin{array}{l}\text { Adh } \\
\text { Adh } \\
\text { Adh } \\
\text { Adh } \\
\text { Adh } \\
\text { Adh }\end{array}$ & $\begin{array}{l}1,93 \\
1,62 \\
1,69 \\
1,54 \\
1,81 \\
1,64\end{array}$ & $\begin{array}{l}1,81 \\
1,76 \\
1,60 \\
1,52 \\
1,80 \\
1,65\end{array}$ & $\begin{array}{l}- \\
- \\
- \\
-\end{array}$ & $\begin{array}{r}91 \\
34 \\
62 \\
110 \\
83 \\
39\end{array}$ & $\begin{array}{l}68 \\
21 \\
63 \\
89 \\
92 \\
55\end{array}$ & $\begin{array}{l}- \\
- \\
- \\
-\end{array}$ & $\begin{array}{r}159 \\
55 \\
125 \\
199 \\
175 \\
94\end{array}$ & $\begin{array}{l}\text { N.S. } \\
\text { N.S. } \\
\text { N.S. } \\
\text { N.S. } \\
\text { N.S. } \\
\text { N.S. }\end{array}$ \\
\hline $\begin{array}{ll}\text { N.S. } & \text { Résulta } \\
* & : P<0,0 \\
* * * & : P<0,0 \\
\mathbf{N} & : \text { Effectif }\end{array}$ & & & & & & & & & \\
\hline
\end{tabular}


On peut être étonné par les différences des durées moyennes de développement d'une population à l'autre pour un même locus; mais le contexte génétique a toutes chances de varier d'une population à l'autre; en outre, les expériences relatives à chacune de ces populations ont été faites à différentes périodes, avec un contrôle de la température et du milieu rigoureux pour chacune d'elles et par suite pour chaque ségrégation, mais avec de légères variations d'une période à l'autre.

En prenant soin de n'utiliser qu'une seule fois chaque fratrie, sur les 25 comparaisons des durées moyennes de développement des génotypes de $1^{\text {re }}$ génération, toutes statistiquement indépendantes, 5 sont statistiquement significatives au seuil de 5 p. 100, soit 20 p. 100 d'entre elles : l'hypothèse d'égalité globale des durées de développement peut être rejetée pour l'ensemble des ségrégations au niveau 0,0072 , ce qui est haute. ment significatif.

Ces résultats montrent que la durée de développement peut varier d'un génotype à l'autre, mais que selon la ségrégation, l'ordre des génotypes n'est pas le même, de sorte que ces valeurs de durée de développement ne permettent pas un classement systématique des génotypes, et paraissent ne dépendre que du croisement lui-même, traduisant ainsi des interactions distinctes entre le locus et les différents génomes des parents.

Les différences entre les durées moyennes de développement peuvent atteindre dans certains croisements des valeurs absolues proches de 2 jours, par exemple 1,81 jour entre les génotypes hétérozygotes et homozygotes Fast au locus Est-C de la ségrégation numéro 3 de la population SCS1 (tableau 2), ce qui représente plus de 25 p. 100 de la durée de la phase d'émergence de cette ségrégation.

2. Ségrégations bifactorielles : du fait de la discontinuité de la distribution des durées de développement, la prise en considération des seules moyennes entraîne une importante perte d'information.

Nous avons donc préféré aux comparaisons directes des durées moyennes, les tests de contingence : G-test, test d'Haldane-Dawson, et le test de comparaison de matrices de Hilton. Le tableau 3 résume les principaux résultats de 5 ségrégations bifactorielles entre des paires de locus génétiquement indépendants. Chaque descendance $\mathrm{F} 1$ est répartie dans un tableau de contingence génotype $\times$ classe de durée de développement.

Sur 10 comparaisons réalisées, 5 seulement ne présentent aucune différence significative au seuil 5 p. 100 pour aucun des 2 premiers tests (G-test et test d'HaldaneDawson), par conséquent 50 p. 100 des ségrégations au minimum présentent des différences entre les durées de développement des génotypes considérés. Le test d'HaldaneDawson est significatif pour 5 des comparaisons $(7,9,11,13$ et 14), tandis que le G-test ne l'est que pour 3 (7, 9 et 11$)$; le recouvrement partiel des résultats des 2 tests traduit leur différence de puissance. Ces résultats très probants reflètent les fluctuations des fréquences génotypiques au cours de la phase d'émergence.

Les effectifs des classes génotypiques peuvent être présentés d'une autre manière. Pour 2 locus donnés, on peut écrire les effectifs sous forme d'une matrice $3 \times 3$, correspondant à la structure génotypique à 2 locus, pour une classe de durée de développement. La comparaison de ces matrices pour diverses classes de durée de développement permet de tester une autre hypothèse : celle de la stabilité des associations génotypiques entre les 2 locus au cours de la phase d'émergence (tableau 3). 


\section{Tableau 3}

Tests de contingence entre génotype et classe de durée de développement dans 10 ségrégations bifactorielles entre couples de locus indépendants.

Contingency test of genotypes and developmental classes in 10 bifactorial segregation between independent loci.

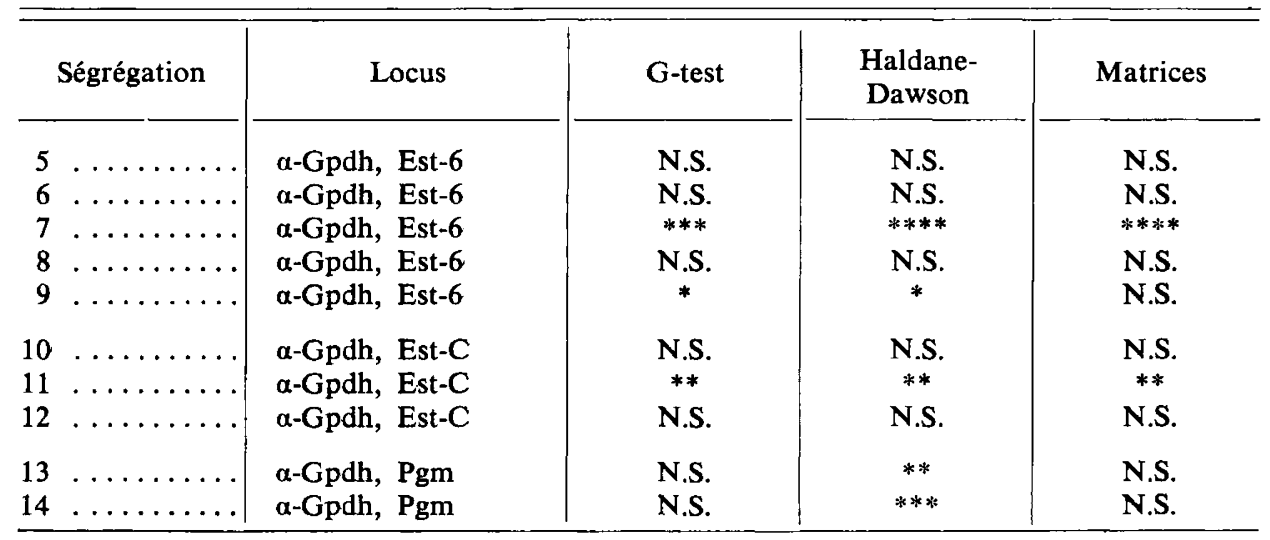

N.S. : Résultat non significatif au seuil $=0,05$.

$$
\begin{array}{ll}
* \quad: & P<0,05 . \\
* * * & : P<0,01 . \\
* * * * & : P<0,001 . \\
* * * * * & : P<10^{-7} .
\end{array}
$$

Les comparaisons 7 et 11 montrent une variation significative des fréquences d'association. Les résultats des ségrégations non indépendantes corroborent les précédents. La seconde des ségrégations non indépendantes est particulièrement intéressante $(E s t-6 / A d h)$ : si l'on n'observe pas de déviation à l'hypothèse de stabilité relative des fréquences génotypiques, on constate par contre une extrême variation des associations entre les 2 locus au cours de la phase d'émergence, ce qui traduit une modification de la composition génétique de la population émergente.

L'étude de ces ségrégations bifactorielles met donc en évidence 2 types de fluctuation qualitativement distinctes : l'une reflète des différences dans la forme de la distribution des émergences de chacun des génotypes, et l'autre, indépendante de la première, reflète les variations des fréquences d'association génotypique entre les 2 locus, au cours de la période d'émergence.

Les différences observées d'un génotype à l'autre apparaissent relativement surprenantes : on pourrait en effet s'attendre à ce que ces différences soient finalement masquées par le reste du génome; or elles ne le sont pas. 


\section{B. Analyse monofactorielle de l'expérience hexafactorielle}

Le tableau 4 représente les durées moyennes de développement de chacun des 3 génotypes pour les 6 locus étudiés ; les analyses de variance ont pu être légitimement réalisées, les distributions d'émergence étant proches de la normalité.

\section{TABLeaU 4}

Valeurs relatives des durées moyennes de développement

des 3 génotypes de première génération et résultat des tests de comparaisons.

Chaque moyenne est suivie d'une erreur standard.

Relative values of mean developmental time of the 3 genotypes in the first generation and comparison of the results. Each mean is followed by its standard error.

\begin{tabular}{|c|c|c|c|c|c|c|c|c|}
\hline \multirow{2}{*}{ Génotypes } & \multicolumn{2}{|c|}{ F. } & \multicolumn{2}{|c|}{ H. } & \multicolumn{2}{|c|}{ S. } & \multicolumn{2}{|c|}{ Résultat des tests } \\
\hline & $\mu$ & $\boldsymbol{\sigma}$ & $\mu$ & $\sigma$ & $\mu$ & $\boldsymbol{\sigma}$ & Test $\mathbf{F}$ & G-test \\
\hline$\alpha-G p d h$ & 4,03 & 0,08 & 3,63 & 0,05 & 3,81 & 0,06 & $* * *$ & $* * * *$ \\
\hline Adh. & 3,86 & 0,06 & 3,60 & 0,05 & 4,02 & 0,08 & $* * *$ & $* * * *$ \\
\hline Est-6 & 3,75 & 0,08 & 3,70 & 0,05 & 3,97 & 0,07 & $* *$ & $* * * *$ \\
\hline Pgm & 3,79 & 0,08 & 3,70 & 0,05 & 3,84 & 0,06 & N.S. & $* * * *$ \\
\hline Est-C & 3,95 & 0,06 & 3,68 & 0,05 & 3,79 & 0,08 & $* *$ & $* * *$ \\
\hline Acph-1 . . & 3,94 & 0,07 & 3,67 & 0,05 & 3,74 & 0,08 & $* *$ & N.S. \\
\hline
\end{tabular}

N.S. : Non significatif au seuil de $=0,05$.

***: : $\mathrm{P}<0,01$.

$* * *: P<0,001$.

$* * * *: P<0,005$.

Pour tous les locus, à l'exception du locus Pgm, les durées moyennes de développement des 3 génotypes sont significativement différentes (test F). Les comparaisons deux à deux des 3 génotypes du locus $P g m$, sont proches du seuil de signification, surtout celle correspondant aux génotypes $H$. et $S$. .

L'observation la plus frappante concernant ces valeurs est la faiblesse systématique des durées de développement des hétérozygotes pour les 6 locus (GIRARD, 1980). Un résultat strictement identique a été observé sur des populations d'huîtres (Zouros et al., 1980).

La durée de développement des hétérozygotes semble donc bien être, quel que soit le locus, plus courte que celle des homozygotes.

L'homogénéité des échantillons des 3 génotypes au cours du temps a été testée par un G-test; les résultats sont hautement significatifs sauf pour le locus $A c p h$, ce qui montre que les distributions de ces génotypes dans le temps sont distinctes les unes des autres.

Les locus Pgm et Acph constituent des cas particuliers qui peuvent s'expliquer, pour le locus $P g m$, par des distributions d'émergences de moyennes proches mais de 
variances légèrement différentes, et pour le locus Acph, par des distributions relativement éloignées de la normalité.

\section{Analyse globale de l'expérience hexafactorielle}

1. Degré d'hétérozygotie et durée de développement.

Dans le tableau 5 ont été mentionnés les effectifs d'individus de $1^{\text {re }}$ génération répartis en fonction des 2 variables aléatoires : durée de développement $D$ et hétérozygotie observée $\mathrm{H}$. Ce tableau a été analysé de la manière suivante :

\section{TABLEAU 5}

Tableau de contingence des individus de première génération de l'expérience hexafactorielle, classés en fonction des 2 variables aléatoires $D$ et $H$.

Contingency table of the first generation individuals in the hexafactorial experiment; they are ordered according to the variables $D$ (developmental time) and $H$ (degree of heterozygosity).

\begin{tabular}{|c|c|c|c|c|c|c|c|c|c|}
\hline $\mathbf{H}$ & & 0 & 1 & 2 & 3 & 4 & 5 & 6 & Somme \\
\hline D & Mâles & 0 & 3 & 4 & 3 & 6 & 3 & 5 & 24 \\
\hline \multirow[t]{2}{*}{1} & Femelles ..... & 0 & 4 & 6 & 8 & 9 & 9 & 5 & 41 \\
\hline & Somme ...... & 0 & 7 & 10 & 11 & 15 & 12 & 10 & 65 \\
\hline \multirow{3}{*}{2} & Mâles & 7 & 17 & 29 & 15 & 28 & 25 & 25 & 146 \\
\hline & Femelles & 7 & 25 & 26 & 28 & 40 & 26 & 12 & 164 \\
\hline & Somme ...... & 14 & 42 & 55 & 43 & 68 & 51 & 37 & 310 \\
\hline \multirow{3}{*}{3} & Mâles $\ldots \ldots \ldots$ & 23 & 41 & 32 & 51 & 52 & 41 & 20 & 260 \\
\hline & Femelles $\ldots \ldots$ & 12 & 33 & 30 & 35 & 46 & 34 & 21 & 211 \\
\hline & Somme ....... & 35 & 74 & 62 & 86 & 98 & 75 & 41 & 471 \\
\hline \multirow{3}{*}{4} & Mâles & 20 & 33 & 42 & 44 & 42 & 31 & 23 & 235 \\
\hline & Femelles & 17 & 28 & 42 & 46 & 50 & 34 & 17 & 234 \\
\hline & Somme ....... & 37 & 61 & 84 & 90 & 92 & 65 & 40 & 469 \\
\hline \multirow{3}{*}{5} & Mâles $\ldots \ldots \ldots$ & 18 & 31 & 16 & 35 & 28 & 21 & 13 & 162 \\
\hline & Femelles & 10 & 26 & 28 & 26 & 32 & 24 & 9 & 155 \\
\hline & Somme ...... & 28 & 57 & 44 & 61 & 60 & 45 & 22 & 317 \\
\hline \multirow{3}{*}{6} & Mâles .. & 10 & 16 & 15 & 17 & 8 & 11 & 3 & 80 \\
\hline & Femelles & 9 & 11 & 18 & 11 & 6 & 20 & 4 & 79 \\
\hline & Somme ....... & 19 & 27 & 33 & 28 & 14 & 31 & 7 & 159 \\
\hline \multirow{3}{*}{8} & Mâles . & 5 & 5 & 1 & 4 & 3 & 5 & 2 & 25 \\
\hline & Femelles & 11 & 6 & 4 & 5 & 9 & 7 & 1 & 43 \\
\hline & Somme ....... & 16 & 11 & 5 & 9 & 12 & 12 & 3 & 68 \\
\hline \multirow{3}{*}{ Somme } & Mâles . & 83 & 146 & 130 & 169 & 167 & 137 & 91 & 932 \\
\hline & Femelles & 66 & 133 & 154 & 159 & 192 & 154 & 69 & 927 \\
\hline & Somme $\ldots \ldots$ & 149 & 279 & 293 & 328 & 359 & 291 & 160 & 1859 \\
\hline
\end{tabular}




\section{TABLEAU 6}

Relation entre le moment d'émergence et le degré d'hétérozygotie chez les imagos de première génération dans l'expérience hexafactorielle.

Relation between hatching time and degree of heterozygosity among the first generation adults in the hexafactorial experiment.

\begin{tabular}{l|c|c|c|c|c}
\hline & Effectifs & d.d.1. & G-test & P & C* \\
\cline { 2 - 5 } Mâles ...... & 932 & 36 & 58,16 & $<0,025$ & 0,237 \\
Femelles .... & 927 & 36 & 52,88 & $<0,025$ & 0,239 \\
Somme .... & 1859 & 36 & 83,50 & $<0,001$ & 0,205 \\
\hline
\end{tabular}

$\mathrm{C}^{*}$ : Coefficient de contingence $=\mathrm{G}^{2} /\left(\mathrm{N}+\mathrm{G}^{2}\right)$.

a) Mise en évidence d'une liaison entre les 2 critères de classification : un test d'indépendance a été réalisé, par le G-test ; les résultats sont portés dans le tableau 6. La liaison entre le degré d'hétérozygotie et la durée d'émergence étant hautement significative, sa nature et sa signification biologique ont été recherchées.

La comparaison des 2 tableaux $7 \times 7$ correspondant aux individus des 2 sexes a été réalisée par un test matriciel de HiLton. Il n'y a pas de différences entre mâles et femelles $(P=70$ p. 100), mais le coefficient de contingence total étant plus faible que celui de chacun des 2 sexes séparés, il apparaît que la liaison, quoique voisine chez les mâles et les femelles n'est pas exactement de même nature.

b) Liaison entre la durée moyenne de développement et le degré d'hétérozygotie des individus : les résultats de l'analyse de variance portant sur la durée moyenne de développement $\mathrm{D}$ figurent dans le tableau 7 . Les durées moyennes $D_{\mathbf{H}}$ diffèrent significativement selon le degré d'hétérozygotie $\mathrm{H}$ observé, et augmentent de manière régulière en raison inverse de celui-ci.

\section{TABLEAU 7}

Comparaison des moyennes des moments d'émergence en fonction du niveau d'hétérozygotie.

Comparison of the hatching time means according to the degree of heterozygosity.

\begin{tabular}{|c|c|c|c|c|}
\hline Fluctuation & d.d.l. & Carrés moyens & $\mathbf{F}$ & $\mathbf{P}$ \\
\hline $\begin{array}{l}\text { Inter } \mathbf{H} \ldots \ldots \ldots \ldots \ldots \\
\text { Résiduelle } \ldots \ldots \ldots \ldots \\
\text { Totale } \ldots \ldots \ldots \ldots \ldots\end{array}$ & $\begin{array}{r}6 \\
1852 \\
1858\end{array}$ & $\begin{array}{r}18,03 \\
2,24\end{array}$ & 8,04 & $<0,0005$ \\
\hline
\end{tabular}

\begin{tabular}{c|c|c|c|c|c|c|c|c}
\hline $\mathbf{H}$ & 0 & 1 & 2 & 3 & 4 & 5 & 6 & $\mathrm{~m}$ \\
\hline $\mathrm{D}$ & 4,450 & 3,914 & 3,754 & 3,841 & 3,602 & 3,801 & 3,394 & 3,796 \\
\hline
\end{tabular}




\section{Tableau 8}

Régression de la durée de développement $D$ sur le degré d'hétérozygotie $H$ et analyse de variance.

Regression of the developmental time $D$ on heterozygosity $H$ and analysis of variance.

\begin{tabular}{|c|c|c|c|c|c|}
\hline Fluctuation & $\begin{array}{c}\text { Somme des carrés } \\
\text { des écarts }\end{array}$ & d.d.l. & Test $\mathbf{F}$ & $\mathbf{P}$ & $\mathrm{r}$ \\
\hline Régression linéaire & 57,236 & 1 & 28,95 & & $-0,123$ \\
\hline Ecart à la linéarité & 34,572 & 5 & 3,49 & 0,005 & \\
\hline Résiduelle $\ldots \ldots \ldots \ldots \ldots$ & 3661,312 & 1852 & & & \\
\hline Totale $\ldots \ldots \ldots \ldots \ldots$ & 3753,520 & 1858 & & & \\
\hline
\end{tabular}

2. Forme de la liaison hétérozygotie/durée de développement.

Les 2 variables étant considérées comme quantitatives $(H=(0,1,2, \ldots, 6)$ et $\mathrm{D}=(1,2, \ldots, 7))$, une régression de $\mathrm{D}$ par rapport à $\mathrm{H}$ et une analyse de variance pour tester l'hypothèse de linéarité sont effectuées (tableau 8).

La pente de la droite de régression étant significativement différente de zéro et négative, on peut conclure que la durée de développement diminue avec le taux d'hétérozygotie.

La figure 1 représente la droite de régression obtenue, $\mathrm{D}=-0,106 \mathrm{H}+4,122$, avec les points moyens de chaque abscisse et le centre de gravité.

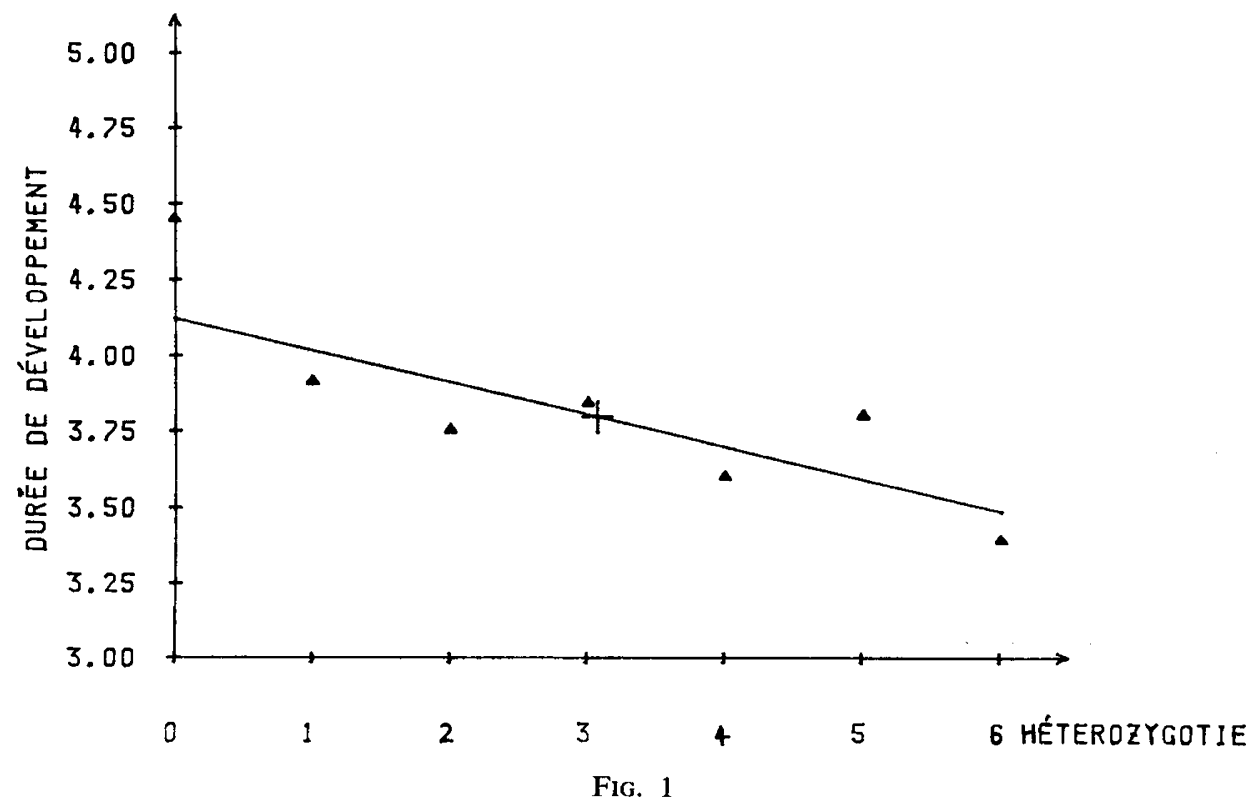

Relation entre l'hétérozygotie moyenne et la durée de développement.

Relation betwcen mean heterozygosity and developmental time. 
3. Etude de la variabilité de $D$ dans chaque classe $H$.

La variabilité de la durée de développement à l'intérieur de chaque classe d'hétérozygotie peut être due à 2 facteurs qualitativement distincts : d'une part, une variabilité intrinsèque de la durée de développement de chaque génotype appartenant à la classe $\mathrm{H}$, d'autre part, une variabilité correspondant au nombre de génotypes différents de la même classe $H$.

Dans un premier temps, une comparaison globale des 7 variances a été réalisée par le test de Bartlett : l'hypothèse d'homogénéité de ces variances peut être rejetée au seuil 0,01 .

L’importance relative du second facteur de variabilité a ensuite été estimée en calculant le nombre de génotypes différents de chaque classe $\mathrm{H}$, et en comparant ces valeurs à celles des variances correspondantes (tableau 9).

\section{TABLeAU 9}

Nombre de génotypes distincts et variance des durées de développement en fonction du nombre de locus à l'état hétérozygote $\boldsymbol{H}$.

Genotypes number and development time variance related to the number of heterozygous loci.

\begin{tabular}{c|c|c|c|c|c|c|c|c}
\hline \hline H & 0 & 1 & 2 & 3 & 4 & 5 & 6 & Somme \\
\cline { 2 - 8 } & & & & & & & & \\
$\begin{array}{r}\text { Nombre de } \\
\text { génotypes . }\end{array}$ & 4 & 24 & 58 & 72 & 46 & 14 & 1 & 219 \\
$\begin{array}{r}\text { Variance de } \\
\mathrm{D} \ldots . . . .\end{array}$ & 2,844 & 2,316 & 2,090 & 2,036 & 2,112 & 2,539 & 2,001 & \\
\hline \hline
\end{tabular}

La variance la plus faible correspond à la classe $H=6$ qui ne contient qu'un seul génotype ; vient ensuite la classe $\mathrm{H}=3$, avec le nombre de génotypes le plus élevé (72) ; la plus grande variance correspond, quant à elle, à la classe $\mathrm{H}=0$ qui ne contient que 4 génotypes.

Ce n'est donc pas le nombre de génotypes différents dans la classe étudiée qui constitue le facteur déterminant de la variabilité totale de la durée de développement.

Ainsi, en dépit de la liaison étroite entre degré d'hétérozygotie et durée moyenne de développement, une grande hétérogénéité subsiste à l'intérieur de chacune des 7 classes d'hétérozygotie, hétérogénéité dont il importera de rechercher plus précisément le déterminisme.

\section{Discussion et conclusion}

L'expérience hexafactorielle permet d'affirmer que la durée de développement est inversement proportionnelle au degré d'hétérozygotie. 
Le déterminisme génétique de la durée de développement, étudié par de nombreux auteurs, n'est pas encore clarifié. Bien que plusieurs gènes majeurs augmentant le temps de développement aient été mis en évidence, les expériences de sélection poursuivies aux alentours des années 50-75, expériences qui utilisaient des souches très variées, n'ont jamais donné de résultats significatifs (SANG \& CLAYTON, 1957 ; Hunter, 1959 ; Clarke et al., 1961 ; SANG, 1962 ; BAKKer, 1969 ; Lints \& GruWez, 1972 et 1974). Cela rend peu probable un déterminisme polygénique spécifique.

Plus récemment, l'étude du polymorphisme enzymatique a montré des différences significatives entre les durées de développement des homozygotes et des hétérozygotes à quelques locus.

DAY et al. (1980) montrent qu'au locus $A d h$, très polymorphe chez Coelopa frigida, les hétérozygotes ont, à l'exception de l'homozygote le plus fréquent dans les populations naturelles, une durée de développement inférieure à celle correspondant aux différents homozygotes.

Van Delden \& Kampling (1979), étudiant le même locus Adh chez Drosophila melanogaster, montrent que les homozygotes SS ont un développement plus lent que les FF ou les FS, de durée moyennes de développement à peu près équivalentes. Marinkovic \& Ayala (1977), comparant 3 souches de Drosophila pseudoobscura pour 3 locus simultanément, démontrent que les triples hétérozygotes ont une durée de développement significativement plus courte que les 2 types d'homozygotes étudiés. Cette influence de l'état hétérozygote sur la diminution de la durée de développement explique l'échec des expériences de sélection, qui augmentent obligatoirement la consanguinité des souches.

Quelques auteurs ont mis en évidence une corrélation entre la vitesse de développement et le degré d'hétérozygotie : Zouros et al. (1980), étudiant des populations d'huîtres ont trouvé un coefficient de corrélation de 0,97 entre les 2 caractères.

Chez la graminée annuelle Liatris cylindrica, SchaAL \& Levin (1976) ont montré une corrélation très forte entre la vitesse de développement et l'hétérozygotie moyenne. L'étude de l'homéostasie du développement par le coefficient d'asymétrie bilatérale de Soule a permis de mettre en évidence de nombreuses corrélations entre l'hétérozygotie des individus et l'homéostasie. Une corrélation négative avec l'asymétrie est toujours trouvée, les individus les plus hétérozygotes ayant une homéostasie plus forte (Soule, 1967 ; Soule \& BAKer, 1968 ; Soule et al., 1973 ; Soule \& YANG, 1974 ; SOULE, 1979).

Les résultats de ce travail sont donc en bon accord avec tous ceux de la littérature, et plusieurs hypothèses explicatives peuvent être envisagées.

La première, classique, est l'exigence d'une coadaptation dans la population d'origine, des gènes considérés. La ségrégation représente alors un processus de destruction des complexes géniques coadaptés, l'expérience hexafactorielle correspondant à une ségrégation extrême - limitée néanmoins par l'absence de crossingover chez les mâles de Drosophila melanogaster - et qui détruit dans la plus large mesure toutes les configurations génotypiques présentes dans les 2 souches d'origine. Le champ de variation des durées de développement observées est d'autant plus important, que certaines structures génotypiques homozygotes coadaptées sont à basses fréquences. Il n'est donc pas étonnant de trouver une dépendance entre la variabilité de la durée de développement de la fratrie et sa structure géno- 
typique. Cependant, cette hypothèse n'explique pas la forme quasi-linéaire de la dépendance, avec une durée de développement systématiquement plus courte chez les hétérozygotes.

La $2^{\mathrm{e}}$ hypothèse est celle d'une over-dominance, l'état hétérozygote étant en luimême responsable d'une diminution de la durée de développement, et l'avantage de cet état reposant sur une flexibilité biochimique due à la présence de 2 molécules distinctes (ou plus dans le cas d'enzymes multimériques) et non plus d'une seule (Haldane, 1954 ; Thoday, 1956 ; Schwartz \& Laughner, 1969 ; Fincham, 1972 ; JoHNSON, 1974 ; SiNGH et al., 1974).

Un individu possédant seulement 2 gènes à l'état hétérozygote peut répondre par 4 stratégies biochimiques distinctes face à une variation des conditions du milieu extérieur, alors que l'homozygote n'a qu'une seule réponse possible.

Au sein de chaque fratrie, les individus les plus hétérozygotes ont une variance des durées de développement faible correspondant probablement à une stabilité plus grande de leur processus de développement.

Ce résultat semble logique sous l'éclairage de la précédente hypothèse, l'hétérozygote ayant plus de manières différentes de compenser les déséquilibres de son développement face aux variations de milieu.

Zouros et al. (1980) ont trouvé un résultat similaire en comparant la variance de l'âge moyen des individus et le degré d'hétérozygotie dans les populations d'huîtres.

Si nous comparons la durée de développement moyenne de la population formée par la descendance $F 1$ analysée dans l'expérience hexafactorielle ( $\mathrm{T}=12$ jours) à la durée moyenne de la population naturelle d'origine, Remoulins 1976 ( $T=16$ jours), nous constatons que le taux moyen d'hétérozygotie très élevé (égal à 50 p. 100, cf. tableau 5) de la population expérimentale diminue la durée de développement de façon très significative.

La vitesse de développement est donc augmentée dans des proportions bien supérieures à celles qui seraient atteintes par l'ensemble des configurations génotypiques homozygotes optimales pour ce caractère.

Cependant, au sein d'une fratrie, la durée de développement d'un individu est d'autant plus courte que l'individu est plus hétérozygote ; mais la variabilité des durées de développement de l'ensemble des individus de la fratrie est d'autant plus grande que l'hétérozygotie moyenne est plus élevée. Ce que Mitron (1978) exprime clairement : "dans chaque population la variation phénotypique la plus faible se trouve chez les individus les plus hétérozygotes, mais les populations ayant une grande diversité de génotypes, leur variance phénotypique doit être élevée ».

Le nombre de travaux en accord avec l'hypothèse d'over-dominance est maintenant fort élevé ; mais la distinction entre les 2 hypothèses de coadaptation et d'over-dominance paraît bien difficile dans la mesure où l'on ne peut apprécier séparément l'influence de chaque gène sur une composante de la viabilité en observant des génotypes qui représentent déjà des structures coadaptées.

Enfin, il faut insister sur les conditions dans lesquelles les expériences ont été réalisées : l'utilisation d'un milieu optimum non compétitif permet d'éliminer l'hypothèse de perturbations dues à une action sélective du milieu sur certains génotypes. 
L'homéostasie génétique (LERNER, 1954) due à la flexibilité biochimique des hétérozygotes peut expliquer les résultats observés ici. Mais l'hypothèse alternative selon laquelle l'avantage observé ne serait pas directement lié à l'over-dominance des locus enzymatiques mais correspondrait à l'association de nos marqueurs avec des gènes responsables d'un développement rapide ne peut être totalement écartée. Elle semble cependant plus difficile à soutenir du fait que toutes les tentatives de sélection pour un développement rapide ont échoué.

La vitesse de développement serait donc conditionnée par la flexibilité du métabolisme général.

Reçu le 5 mars 1985.

Accepté le 3 mars 1986.

\section{Remerciements}

Je tiens à remercier $M^{\text {me }}$ Claudine Petit et $M$. Jean-Michel Goux pour leur aide critique et leur suggestions, et $\mathbf{M}^{\mathrm{me}}$ Monique LeHMaNn dont l'assistance technique m'a été très précieuse.

Cet article est présenté dans le cadre de l'U.A. 693 et du G.R.E.C.O. 44 du C.N.R.S.

\section{Références bibliographiques}

BAKKER K., 1969. Selection for rate of growth and its influence on competitive ability of larvae of Drosophila melanogaster. Nether. J. Zool., 19, 541-595.

Beckman L., Johnson F.M., 1964. Esterases variations in Drosophila melanogaster. Hereditas, 51, $212-220$.

Boesiger E., 1962. Sur le degré d'hétérozygotie des populations naturelles de Drosophila melanogaster et son maintien par la sélection sexuelle. Bull. Biol., 96 (1), 1-121.

Chaisson R.E., Serunian L.A., Schopf T.J.M., 1976. Allozyme variation between two marshes and possible heterozygote superiority within a marsh in bivalve Modoilus demissus. Biol. Bull., 151, 404.

Clarke J.M., Maynard Smith J., Sondhi K.C., 1961. Asymmetrical response to selection for rate of development in Drosophila subsobscura. Genet. Res., 2, 70-81.

DaGnelie P., 1975. Théorie et Méthodes statistiques, 463 pp., Les Presses Agronomiques de Gembloux, Gembloux.

Day T.H., Dobson T., Hillier P.C., Parkin D.T., Clarke B., 1980. Different rates of development associated with the alcohol dehydrogenase locus in the seaweed fly, Coelopa frigida. Heredity, 44, 321-326.

DobZhansky Th., 1937. Genetics and the origin of species, 364 pp. Columbia University Press, New York.

Fincham J.R.S., 1972. Heterozygous advantage as a likely general basis for enzyme polymorphisms. Heredity, 28, 387-391.

FISHER R.A., 1949. The theory of inbreeding. 150 pp., Oliver and Boyd, Edinburgh and London.

GIRARD P., 1976. Etude du polymorphisme enzymatique de quinze populations naturelles françaises de Drosophila melanogaster, $38 \mathrm{pp}$. Thèse de troisième cycle, Université de Paris 7.

GIRARD P., 1980. Distortion of mendelian segregation in enzymatic polymorphism of Drosophila melanogaster. Genetika, 12 (1), 57-69. 
Girard P., Palabost L., Petit C., 1977. Enzymatic variation at seven loci in nine natural populations of Drosophila melanogaster. Biochem. Genet., 15, 589-599.

GRELL E.H., 1967. Electrophoretic Variants of $\alpha$-Glycerophosphate Dehydrogenase in Drosophila melanogaster. Science, 158, 1319-1320.

Grell E.H., JAcobson K.B., MURPhy J.B., 1965. Alcohol Dehydrogenase in Drosophila melanogaster. Science, 149, 80-82.

Haldane J.B.S., 1954. The Statics of Evolution. In : Huxley J., Hardy A.C., Ford E.B. (ed.), Evolution as a Process, 109-121. Allen and Unnin, London.

HrLton G., 1957. An Algorithm for Detecting Differences between Transition Probability Matrices. Miscellanea, 5, 80-86.

HJoRTh J.P., 1970. A Phosphoglucomutase locus in Drosophila melanogaster. Hereditas, 64, 146-148.

HUNTER P.E., 1959. Selection of Drosophila melanogaster for length of larval period. Zeitschrift für Vererbungslehre, 90, 7-28.

JohNson G.B., 1974. Enzyme polymorsphism and metabolism. Science, 184, 28-37.

LERNER I.M., 1954. Genetic Homeostasis. 134 pp., Oliver and Boyd, London.

LEwontin R.C., 1965. The genetics of Colonizing Species. 322 pp., Baker H.G. \& Ledyard Stebbins, New York.

Lewontin R.C., 1974. The Genetic Basis of Evolutionary Change, 346 pp., Columbia University Press, New York.

Lints F.A., Gruwez G., 1972. What determines the duration of development in Drosophila melanogaster. Mech. Age. Dev., 1, 285-297.

Lints F.A., Gruwez G., 1974. Déterminisme de la durée de développement chez Drosophila melanogaster. Ann. Génét. Sél. Anim., 6 (1), 103-116.

MAC INTYRE R.J., 1966. The genetics of an acid phosphatase in Drosophila melanogaster and Drosophila simulans. Genetics, 53, 461-474.

Marinkovic D., Ayala F.J., 1977. Fitness of allozyme variants in Drosophila pseudoobscura iii : Possible factors contributing to the maintenance of polymorphism in nature. Genetica, 47 (1), 65-70.

Mrtron J.B., 1978. Relationship between heterozygosity for enzyme loci and variation of morphological characters in natural populations. Nature, 273, 661-662.

Mitton J.B., Grant M.C., 1980. Observations on the Ecology and Evolution of Quaking aspen, Populus tremuloides, in the Colorado front range. Amer. J. Bot., 67 (2), 202-209.

SANG J.H., 1962. Selection for rate of larval development using Drosophila melanogaster cultured axenically on different diets. Genet. Res., 3, 90-109.

Sang J.H., Clayton G.A., 1957. Selection for larval development time in Drosophila. J. Heredity, 48, 265-270.

SchaAl B.A., Levin D.A., 1976. The demographic genetics of Liatria cylindracea Michx (Compositae). Amer. Natur., 110, 191-206.

Schwartz D., Laughner W.J., 1969. A molecular basis for heterosis. Science, 166, 626-627.

Singh R.S., HUbby J.L., Lewontin R.C., 1974. Molecular Heterosis for Heat-Sensitive Enzyme Alleles. Proc. Nat. Acad. Sci., U.S.A., 71 (5), 1808-1810.

SingH S.M., Zouros E., 1978. Genetic variation associated with growth rate in the American oyster (Crassostrea virginica). Evolution, 32 (2), 342-353.

Soule M.E., 1967. Phenetics of natural populations. II. Asymmetry and evolution in a lizard. Amer. Natur., 101, 141-160.

Soule M.E., 1979. Heterozygosity and Developmental Stability : another look. Evolution, 33 (1), 396-401.

Soule M.E., BaKer B., 1968. Phenetics of natural populations. IV. - The population asymmetry parameter in the butterfly Coenonympha tullia. Heredity, 23, 611-614.

Soule M.E., Yang S.Y., 1974. Genetic variation in side-blotched lizards on islands in the Gulf of California. Evolution, 27, 593-600.

Soule M.E., Yang S.Y., Weiler M.G.W., Gorman G.C., 1973. Island Lizards : the Genetic-Phenetic Variation Correlation. Nature, 242, 191-193. 
Stalker H.D., 1976. Enzymes and reproduction in natural populations of Drosophila euronotus. Genetics, 84, 375-384.

Sved J.A., Ayala F.J., 1970. A population cage for heterosis in Drosophila pseudoobscura. Genetics, 66, 97-113.

SVEd J.A., ReEd T.E., Bodmer W.F., 1967. The number of balanced polymorphisms that can be maintained in a natural population. Genetics, 55, 469-481.

Teissier G., 1942. Vitalité et fécondité relatives de diverses combinaisons génétiques comportant un gène léthal, chez la Drosophile. C.R. Acad. Sci., 214, 241-244.

ThoDAY J.M., 1956. Balance, Heterozygosity and Developmental Stability. Cold. Spring Harb. Symp. Quant. Biol., 21, 318-326.

Van Delden W., Kampling A., 1979. The alcohol dehydrogenase polymorphism in populations of Drosophila melanogaster. 3. Differences in developmental times. Genet. Res. Camb., 33, 15-27.

Wool.F B., 1956. The Log likelihood ratio test (the G-test). Ann. Hum. Genet., 21, 397-409.

WRIGHT T.R.F., 1963. The genetics of an esterase in Drosophila melanogaster. Genetics, 48, 787-801.

Zouros E., Singh S.M., Miles H.E., 1980. Growth rate in oysters : An overdominant phenotype and its possible explanations. Evolution, 34 (5), 856-867. 